\title{
Fontan operation with a viable and growing conduit using pedicled autologous pericardial roll: Serial changes in conduit geometry
}

Iki Adachi, MD, ${ }^{a}$ Toshikatsu Yagihara, MD, ${ }^{a}$ Koji Kagisaki, MD, ${ }^{a}$ Ikuo Hagino, MD, ${ }^{a}$ Toru Ishizaka, MD, ${ }^{a}$ Masahiro Koh, MD, ${ }^{a}$ Hideki Uemura, MD, ${ }^{b}$ and Soichiro Kitamura, MD ${ }^{a}$

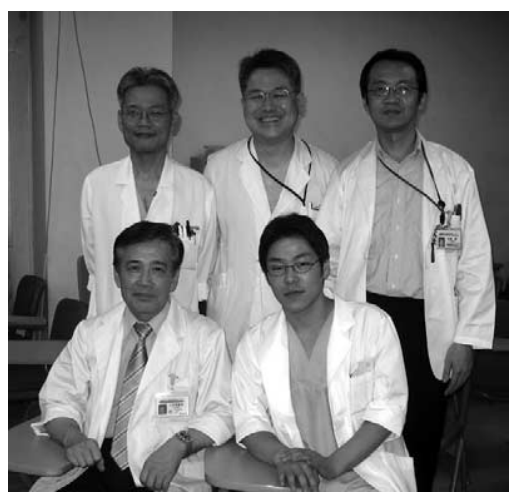

Kagisaki, Hagino, Ishizaka (top, from left) Yagihara, Adachi (bottom, from left)

Supplemental material is available online.
From the Department of Cardiovascular Surgery, ${ }^{\mathrm{a}}$ National Cardiovascular Center, Osaka, Japan, and the Department of CardioThoracic Surgery, ${ }^{\mathrm{b}}$ Royal Brompton Hospital, London, United Kingdom.

Received for publication April 9, 2005; revisions received April 9, 2005; accepted for publication April 9, 2005.

Address for reprints: Toshikatsu Yagihara, MD, 5-7-1 Fujishiro-dai, Suita, Osaka 5658565, Japan (E-mail: yagihara@hsp.ncvc. go.jp).

J Thorac Cardiovasc Surg 2005;130:1517-22

$0022-5223 / \$ 30.00$

Copyright (C) 2005 by The American Association for Thoracic Surgery

doi:10.1016/j.jtcvs.2005.07.050
Objective: We sought to examine midterm results of the Fontan operation with an extracardiac conduit using pedicled autologous pericardial roll, with special attention to angiographic evaluation of serial changes in conduit geometry.

Methods: Of 202 patients subjected to the Fontan operation since 1996, the conduit was used in 28 patients who had intact pericardium. We retrospectively reviewed a consecutive series of these patients, and serial changes in conduit geometry were accessed in 16 patients who underwent catheter examinations twice at $1.1 \pm 0.4$ and $4.5 \pm 1.4$ years postoperatively.

Results: There was one early and one late death. Except for a patient with apicocaval juxtaposition in whom a conduit occlusion developed caused by compression between the vertebral bodies and the ventricle, there were no important complications. Angiographically, conduit volume, estimated by using the MULTI-SLICE method, increased significantly $\left(3490 \pm 2166\right.$ to $\left.5426 \pm 3081 \mathrm{~mm}^{3}, P<.001\right)$, whereas the volume per body weight remained unchanged. Conduit diameter increased significantly at both the inferior vena caval end $(16.8 \pm 4.8$ to $19.8 \pm 4.8$ $\mathrm{mm}, P<.001)$ and the pulmonary artery end $(11.9 \pm 3.8$ to $14.2 \pm 4.2 \mathrm{~mm}, P<$ .001 ), whereas the diameter indexed to the normal right pulmonary artery remained unchanged. The cross-sectional area of the conduit increased in parallel with that of the normal right pulmonary artery. The ratio of the widest to the narrowest diameter of the conduit exhibited no significant change.

Conclusions: Midterm results of the Fontan operation with pedicled autologous pericardial roll were favorable. Proportional increase of conduit size was demonstrated, with its shape preserved. This suggested a potential of the conduit to grow and that growth correlated with somatic development.

$\mathrm{S}$ urgical repair with native tissue has widened the indications of congenital cardiovascular surgery and has gained increasingly widespread application to various reconstructive procedures. Autologous pericardium has been used for construction of a cavopulmonary connection in the Fontan operation, with the expectation of possible growth ability and less thrombogenicity of the conduit. ${ }^{1-6}$ Although favorable midterm outcomes have been reported, ${ }^{7}$ no clinical study has quantitatively evaluated serial changes in conduit shape and size. Our objective was to examine midterm results of the Fontan operation using a pedicled pericardial conduit, with special attention to angiographic evaluation of serial changes in conduit geometry.

\section{Patients and Methods}

\section{Patient Population}

Since 1996, we have used the Fontan operation with an extracardiac conduit constructed with pedicled autologous pericardial roll (PAPR) in patients with an intact pericardium. Of 202 


\author{
Abbreviations and Acronyms \\ IVC = inferior vena cava \\ $\mathrm{PA}=$ pulmonary artery \\ PAPR $=$ pedicled autologous pericardial roll \\ $\mathrm{SVC}=$ superior vena cava
}

patients subjected to the Fontan operation between January 1996 and February 2005, a PAPR conduit was used in 28 patients. The median age at operation was 1.3 years (range, $0.8-19.7$ years), and median body weight was $8.5 \mathrm{~kg}$ (range, $4.6-51.3 \mathrm{~kg}$ ). The primary diagnosis was tricuspid atresia in 10 , unbalanced common atrioventricular canal in 5, double-inlet left ventricle in 4 , atrioventricular discordance with double-outlet right ventricle in 2 and with hypoplastic right ventricle in 1, pulmonary atresia with intact ventricular septum in 3 , mitral atresia in 1, double-inlet right ventricle in 1 , and double-outlet right ventricle with noncommitted ventricular septal defect in 1. Atrial arrangement was situs solitus in 22, right isomerism in 4 , and situs inversus in 2 . Concomitant procedures were as follows: pulmonary artery (PA) plasty in 8; enlargement of atrial septal defect, ventricular septal defect, or both in 6; additional aortopulmonary anastomosis in 2; mitral valve plasty in 1 ; and cryoablation of the right atrium in 1 . Of the 28 patients, 19 had previously undergone palliative operations, and 2 had a history of median sternotomy.

Information regarding the late status of patients was obtained from medical records. Median duration of follow-up was 5.5 years (range, 1.2-8.4 years). All surviving patients have been followed up postoperatively in our outpatient clinic.

\section{Surgical Procedure}

The chest was opened through a median sternotomy, and the pleural space of the corresponding side was opened widely. A sufficiently large rectangular flap of pericardium was cut, leaving it pedicled to preserve its vascular connections, with care taken not to injure the phrenic nerve. The flap was then rolled into a tube shape with a running suture of 6-0 polypropylene with minimal tension and relatively rough pitch to maintain the blood supply to PAPR (Figure 1, $A$ ). Intraoperative measurements of PAPR diameter in the 28 patients were $16 \mathrm{~mm}$ in $2,17 \mathrm{~mm}$ in $2,18 \mathrm{~mm}$ in 12 , $19 \mathrm{~mm}$ in $1,20 \mathrm{~mm}$ in $8,22 \mathrm{~mm}$ in 2 , and $26 \mathrm{~mm}$ in 1 . The length of the PAPR conduit was determined on the basis of the distance between the inferior vena cava (IVC) and the PA.

Subsequent to injection of $0.15 \mathrm{~mL} / \mathrm{kg}$ heparin, the superior vena cava (SVC) was connected to the upper surface of the PA by means of bidirectional Glenn anastomosis in all but one patient, who had undergone bidirectional Glenn anastomosis previously. In 19 patients a temporary bypass from the SVC to the atrium was used when cross-clamping the SVC, whereas in the other 9 patients cardiopulmonary bypass was used because of either intracardiac procedures or extended PA plasty. The temporary bypass was a circuit placed between the caval veins and the atrium for venous drainage, and it consisted of 2 cannulae and a short connector with a device for air trapping. ${ }^{8}$ The PAPR conduit was anastomosed to the lower surface of the PA by using a temporary bypass from the IVC to the atrium in cases without cardiopulmonary bypass. The

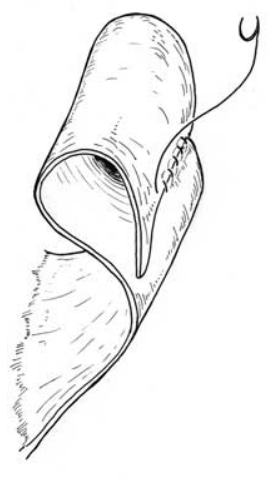

A

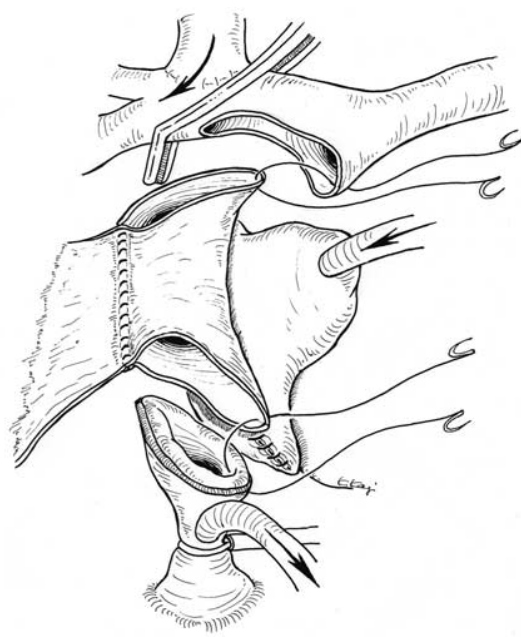

B
Figure 1. A, A sufficiently large rectangular flap of pericardium was cut, leaving it pedicled so as to preserve its vascular connections, and the flap was then rolled into a tube shape. B, The schema of extracardiac grafting with a pedicled autologous pericardial roll (PAPR) conduit with the aid of a temporary bypass from the inferior vena cava (IVC) to the atrium. The left pulmonary artery was clamped to control retrograde blood return (not depicted for simplicity). The venoatrial junction was divided obliquely to obtain an adequate orifice for anastomosis between a PAPR conduit and the IVC, leaving a small sleeve of atrial musculature around the IVC.

venoatrial junction was divided obliquely to obtain an adequate orifice for anastomosis between the PAPR conduit and the IVC, leaving a small sleeve of atrial musculature around the IVC. The proximal stump of the atrial side was oversewn, and the distal stump of the IVC side was anastomosed to the PAPR conduit, which had been trimmed and tailored (Figure 1, B). In 2 patients a fenestration was placed by interposing a 5-mm Gore-Tex tube graft between the PAPR conduit and the atrium because of relatively high pulmonary vascular resistance $\left(3.0\right.$ and $2.8 \mu \mathrm{m}^{2}$, respectively). In addition to routine monitoring of SVC pressure through a percutaneously placed catheter, femoral venous pressure was confirmed not to exceed $20 \mathrm{~mm} \mathrm{Hg}$ during IVC clamping in cases without cardiopulmonary bypass.

\section{Anticoagulation}

Anticoagulation therapy with warfarin sodium and dipyridamole was continued until 1 year postoperatively. The international normalized ratio was maintained at around 2.0. The duration of dipyridamole administration was determined by the patient's personal pediatrician, although indefinite continuation of dipyridamole was recommended when compliance could be predicted and there were no obvious contraindications.

\section{Angiographic Evaluation}

Of the 28 patients with the PAPR conduit, 16 underwent catheter examinations twice at $1.1 \pm 0.4$ and $4.5 \pm 1.4$ years postopera- 
tively. During this period, their body weight and body surface area increased significantly (body weight: $12.4 \pm 2.6$ to $19.1 \pm 3.4 \mathrm{~kg}$, $P<.001$; body surface area: $0.5 \pm 0.1$ to $\left.0.8 \pm 0.1 \mathrm{~m}^{2}, P<.001\right)$. PAPR diameter, cross-sectional area, and volume were measured, and serial changes of these parameters were compared with somatic growth. As control subjects, 18 patients with an artificial graft as an extracardiac conduit who underwent postoperative catheter studies twice were evaluated. Graft volume was estimated by using the MULTI-SLICE method, which is usually used for ventricular volumetry. Each graft was traced in both frontal and lateral views, and the selected structure was divided into 32 crosssections (Figure E1). Finally, cross-sectional areas of each slice were integrated to calculate graft volume. The ratio of the widest to the narrowest diameter of the PAPR conduit was estimated in either the frontal or lateral view to determine proportional change in the PAPR conduit (Figure E2). Three individuals (I.A., T.Y., and K.K.) determined the measurement points individually to eliminate subjectivity of examiner measurements. Furthermore, all procedures of measurement, such as diameter calculation and graft tracing, were repeated 3 times in each case to minimize potential sources of error from manual maneuvers, and mean values of the 3 measurements were used as final discrete values. All angiographic measurements were performed with DCM View3 angiogram software (Climb Medical System).

\section{Statistical Analysis}

Means and standard deviations were calculated for continuous variables. Comparisons were made between values obtained postoperatively at the 2 angiographies by using a paired $t$ test. All analyses were performed with StatView statistical software (Abacus Concepts).

\section{Results}

\section{Mortality and Morbidity}

There was 1 (3.6\%; 95\% confidence interval, $0.6 \%-17.7 \%)$ early death caused by cerebral infarction. Because the patient had no fenestration and was hemodynamically stable after the operation, embolic infarction during cardiopulmonary bypass was suspected. There was 1 late death 18 months after the Fontan operation caused by viral pneumonia. Although postmortem examination was not performed for this patient, echocardiography revealed no cardiac or conduit-related problems. Except for a patient with apicocaval juxtaposition in whom conduit occlusion developed as a result of compression between the vertebral bodies and the ventricle, there were no important complications. The median durations of mechanical ventilation, intensive care unit stay, and chest tube drainage among hospital survivors were 1 day, 6 days, and 6 days, respectively. Two patients required prolonged chest tube drainage ( $>2$ weeks) but exhibited spontaneous resolution. One patient had phrenic nerve palsy on the side opposite the pericardial harvest, which could be observed conservatively. There was no conduit-related reoperation in any patient except for 1 patient in whom conduit occlusion developed, as mentioned above. Of the 27 patients who survived the Fontan com- pletion, all remain in sinus rhythm, with an adequate rate at their latest follow-up without prescription of antiarrhythmic drugs. Patients were otherwise free of any other late complications.

\section{Angiographic Evaluation}

PAPR volume increased significantly (3490 \pm 2166 to $5426 \pm$ $\left.3081 \mathrm{~mm}^{3}, P<.001\right)$, whereas artificial graft volume decreased significantly $\left(6210 \pm 2795\right.$ to $5853 \pm 2481 \mathrm{~mm}^{3}, P$ $=.026$ ). PAPR volume per body weight remained unchanged ( $286 \pm 173$ to $\left.282 \pm 155 \mathrm{~mm}^{3} / \mathrm{kg}, P=.775\right)$. PAPR diameter increased significantly at both the IVC and PA ends (IVC: $16.8 \pm 4.8$ to $19.8 \pm 4.8 \mathrm{~mm}, P<.001$; PA: $11.9 \pm 3.8$ to $14.2 \pm 4.2 \mathrm{~mm}, P<.001)$. However, when PAPR diameter was indexed to normal right PA diameter, it remained unchanged at both the IVC and PA ends (IVC: $162 \% \pm 46 \%$ to $160 \% \pm 35 \%, P=.722$; PA: $114 \% \pm 36 \%$ to $114 \% \pm 31 \%, P=.963)$. The ratio of the widest to the narrowest diameter of the PAPR conduit exhibited no significant change in either the frontal or lateral view (frontal: $2.0 \pm 0.8$ to $1.9 \pm 0.7, P=.495$; lateral: $1.9 \pm 0.7$ to 2.0 $\pm 0.6, P=.759$; Figure 2 ). The cross-sectional area of the narrowest portion of the PAPR conduit increased in parallel with that of the normal right PA (Figure 3). Neither aneurysmal change nor stenosis of the PAPR conduit was observed, and no pressure gradient was detected across the PAPR conduit in any patient except for 1 patient in whom conduit occlusion developed.

\section{Discussion}

Since Fontan and Baudet ${ }^{9}$ described the first successful right-sided heart bypass operation in patients with singleventricle physiology, several modifications have been developed to obtain better quality of circulation and ease of surgical procedures. Of such modifications, the extracardiac Fontan operation has been widely accepted as a technique of choice because of its several advantages, although concern remains regarding the risk of thrombosis and lack of growth ability of the artificial graft in the systemic venous pathway. In 1992, Hvass and colleagues ${ }^{1}$ first reported their successful use of a pedicled pericardial tube for construction of a Fontan pathway, and similar reports have since been published, ${ }^{2-6}$ with midterm results that appear to be equivalent to those of the extracardiac Fontan operation with an artificial graft. ${ }^{7}$ Pathway connection with autologous pericardium might have additional potential advantages over that with an artificial graft. Of such advantages, growth potential is theoretically a significant one because Fontan completion has recently been performed much earlier. ${ }^{10}$ In 1997, Gundry and associates ${ }^{4}$ described in their report that serial follow-up echocardiograms showed continued growth of the extracardiac lateral tunnels constructed with pedicled pericardium in length and width. However, no previous clinical 

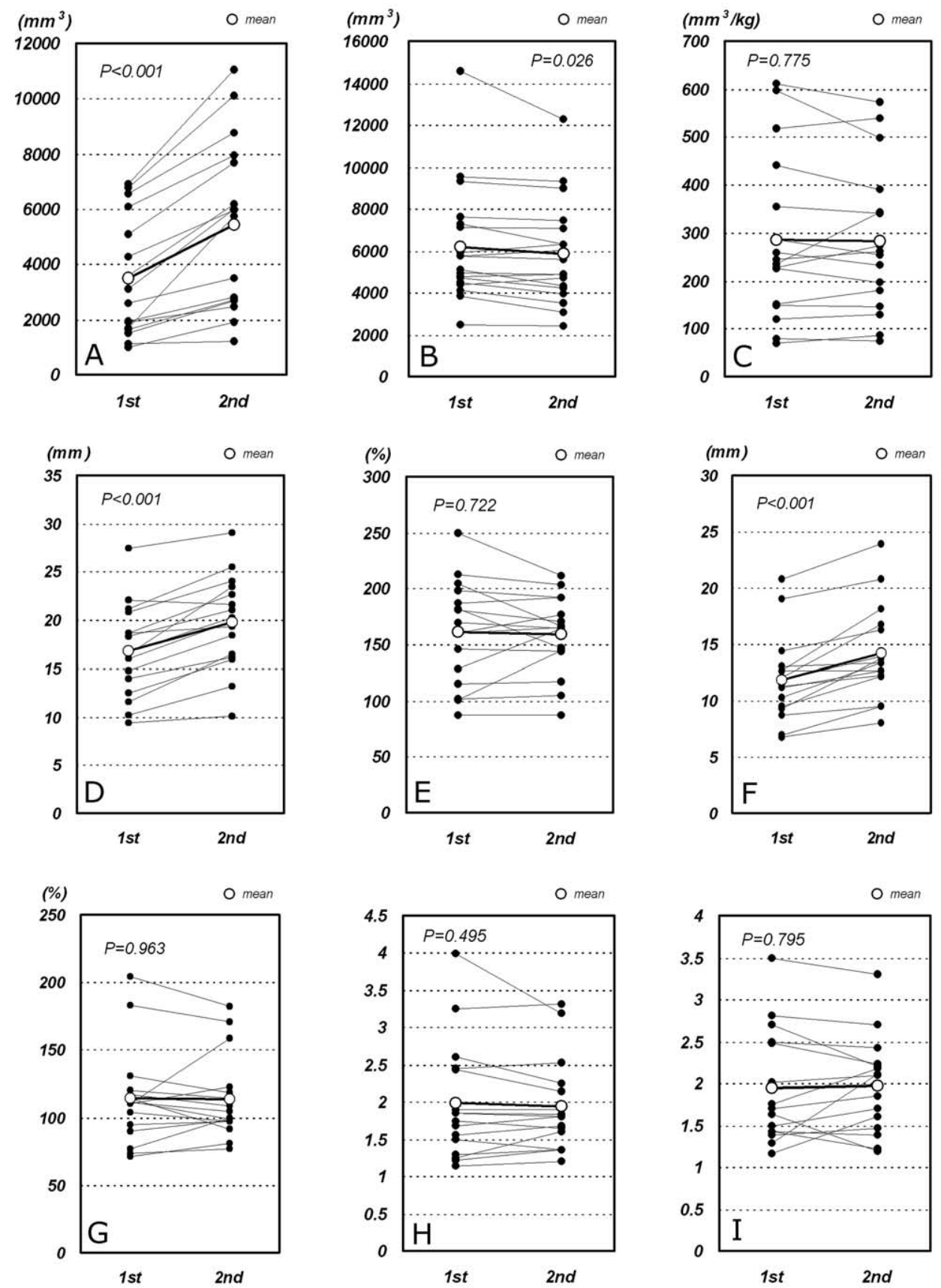

Figure 2. Serial changes in pedicled autologous pericardial roll (PAPR) volume (A), artificial graft volume (B), PAPR volume indexed to body weight (C), PAPR diameter at the inferior vena caval (IVC) end (D), PAPR diameter at the IVC end indexed to normal right pulmonary artery (PA) diameter (E), PAPR diameter at the PA end (F), PAPR diameter at the PA end indexed to normal right PA diameter (G), widest/narrowest ratio in PAPR (frontal view; $H$ ), and widest/narrowest ratio in PAPR (lateral view; I).

study has quantitatively evaluated serial changes in conduit shape and size with time, although the ability of pedicled pericardium to grow has been clearly shown in an experimental animal study. ${ }^{11}$
In our study significant increases in PAPR volume and diameter between the 2 postoperative angiographies were demonstrated quantitatively. However, when these values were indexed to somatic parameters, the indexed values 


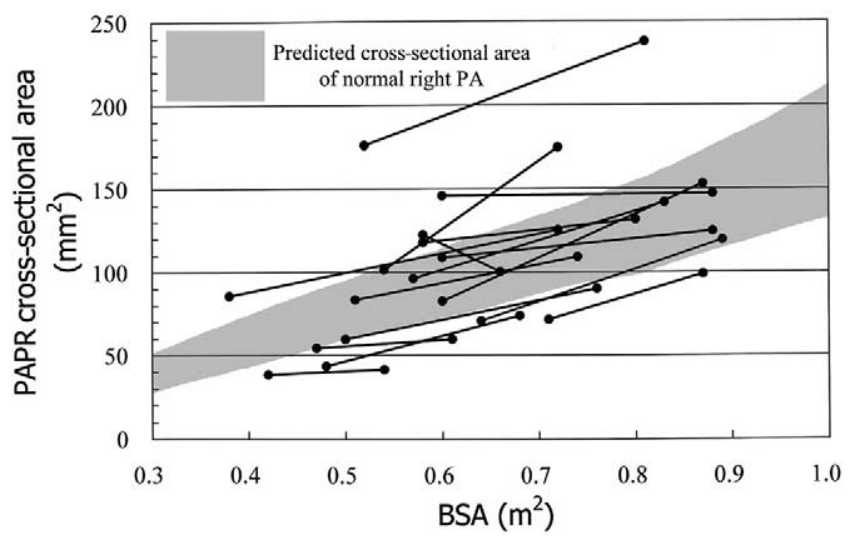

Figure 3. Relation between cross-sectional area of pedicled autologous pericardial roll (PAPR) and body surface area (BSA). PA, Pulmonary artery.

remained unchanged. The cross-sectional area of the PAPR conduit increased nearly in parallel with that of the normal right PA. These findings indicated that a significant increase in PAPR size was correlated with somatic development. Furthermore, invariability of PAPR proportion was demonstrated, although in previous studies use of autologous pericardium as an implant for vascular substitute yielded mixed results, including shrinkage caused by fibrosis with retraction or thinning with dilatation. ${ }^{12}$ Despite the difficulty in clearly distinguishing between growth and mere dilatation, the potential of the PAPR conduit to grow was suggested by the proportional increase in conduit size with preservation of shape.

Some discrepancy was found in PAPR conduit diameters between angiographic examination and intraoperative findings. The angiographic mean value of the PA end diameter at the first catheter examination $(11.9 \pm 3.8 \mathrm{~mm})$ was $36 \%$ less than the intraoperative measurement $(18.6 \pm 1.3 \mathrm{~mm})$. This phenomenon can be regarded as a result of pericardial remodeling rather than shrinkage because a significant increase in diameter was demonstrated over time. In other words, we might observe the intrinsic adjustability of the PAPR conduit, which has the ability to vary its own shape in response to the flow amount passing through it.

The obvious disadvantage of this modification is its limited application. In fact, we could use the technique in less than 14\% (28/202) of patients undergoing Fontan completion over the 9-year period of this study because of the lack of intact pericardium. There were no other anatomic limitations that prevented us from using this technique in the majority of our patients undergoing the Fontan operation. However, apicocaval juxtaposition might be consid- ered as a contraindication of this technique because PAPR was more vulnerable to external compression than a rigid artificial graft.

A potential disadvantage of the geometric approach to measuring graft volume on angiographic studies is possible variability in calculated volume from observer to observer when the graft region of interest is drawn manually or when the anastomotic lines of the graft are defined manually. This is a major problem in the type of angiographic examinations performed in the present study because of the inherently overly smooth anastomotic lines resulting from construction of pathways using only native tissue. Three individuals determined the measurement points individually in the present study to eliminate subjectivity of examiner measurement, and as a result, there was little interobserver variation. Furthermore, little intraobserver variability was noted, although all procedures of measurement, such as diameter calculation and graft tracing, were repeated 3 times in each case to minimize potential sources of error from manual maneuvers.

In conclusion, midterm results of the Fontan operation with a PAPR conduit were favorable. Proportional increase of conduit size was demonstrated, with its shape preserved. This suggests a potential of the conduit to grow and that growth correlated with somatic development.

We wish to express our appreciation for the excellent technical assistance of Akiko Kada (General Clinical Research Unit, National Cardiovascular Center, Japan) in the statistical analysis.

\section{References}

1. Hvass U, Pansard Y, Bohm G, Depoix JP, Enguerrand D, Worms AM. Bicaval pulmonary connection in tricuspid atresia using an extracardiac tube of autologous pediculated pericardium to bridge inferior vena cava. Eur J Cardiothorac Surg. 1992;6:49-51.

2. Hashimoto K, Kurosawa H, Tanaka K, Yamagishi M, Koyanagi K, Ishii S, et al. Total cavopulmonary connection without the use of prosthetic material: technical considerations and hemodynamic consequences. J Thorac Cardiovasc Surg. 1995;110:625-32.

3. van Son JA, Reddy M, Hanley FL. Extracardiac modification of the Fontan operation without use of prosthetic material. J Thorac Cardiovasc Surg. 1995;110:1766-8.

4. Gundry SR, Razzouk AJ, del Rio MJ, Shirali G, Bailey LL. The optimal Fontan connection: a growing extracardiac lateral tunnel with pedicled pericardium. J Thorac Cardiovasc Surg. 1997;114: $552-8$

5. Okabe H, Nagata N, Kaneko Y, Kobayashi J, Kanemoto S, Takaoka T. Extracardiac cavopulmonary connection of Fontan procedure with autologous pedicled pericardium without cardiopulmonary bypass. J Thorac Cardiovasc Surg. 1998;116:1073-5.

6. Uemura H, Yagihara T, Kawahira Y. The extracardiac Fontan procedure using a pedicled pericardial roll without cardiopulmonary bypass. J Thorac Cardiovasc Surg. 1999;117:1046-7.

7. Woods RK, Dyamenahalli U, Duncan BW, Rosenthal GL, Lupinetti FM. Comparison of extracardiac Fontan techniques: pedicled pericardial tunnel versus conduit reconstruction. J Thorac Cardiovasc Surg. 2003;125:465-71

8. Uemura H, Yagihara T, Yamashita K, Ishizaka T, Yoshizumi K, Kawahira Y. Establishment of total cavopulmonary connection with- 
out use of cardiopulmonary bypass. Eur J Cardiothorac Surg. 1998;13:504-7.

9. Fontan F, Baudet E. Surgical repair of tricuspid atresia. Thorax. 1971;3:240-8.

10. Uemura H, Yagihara T, Kawahira Y, Yoshikawa Y, Kitamura S. Total cavopulmonary connection in children with body weight less than 10 kg. Eur J Cardiothorac Surg. 2000;17:543-9.
11. Guyton RA, Dorsey LM, Silberman MS, Hawkins HK, Williams WH, Hatcher CR Jr. The broadly based pericardial flap. A tissue for atrial wall replacement that grows. J Thorac Cardiovasc Surg. 1984;87:619-25.

12. Cheung DT, Choo SJ, Grobe AC, Marchion DC, Luo HH, Pang DC, et al. Behavior of vital and killed autologous pericardium in the descending aorta of sheep. J Thorac Cardiovasc Surg. 1999;118: 998-1005.

\section{The Journal of Thoracic and Cardiovascular Surgery Conflict of Interest Policy}

To assure fairness to authors submitting work for consideration in The Journal of Thoracic and Cardiovascular Surgery, a mechanism exists for managing conflicts of interest. The editor and each of the section editors complete a "Conflict of Interest" form that identifies any and all relationships with commercial and other academic entities. When the editor has a potential conflict because of a relationship with another entity or author, the editor appoints an alternate editor from among the section editors or editorial board members who assumes the entire responsibility for final decisions on the manuscript in question. The editor does not read the reviews that are submitted nor engage in discussing the manuscript prior to the final decision. When the conflict of interest involves a section editor, a "guest section editor" is appointed who fills the role normally played by the conflicted section editor. All members of the editorial board and reviewers are asked to indicate any conflict of interest when they agree to review a manuscript. 

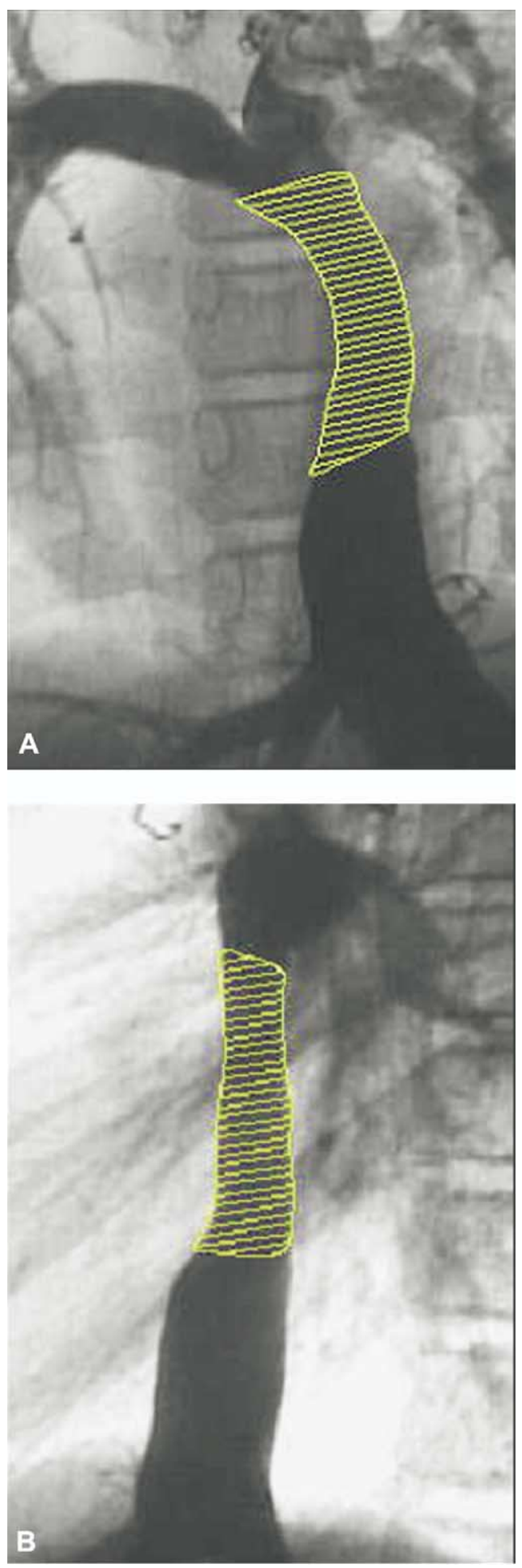

Figure E1. Each graft was traced in both frontal (A) and lateral (B) views, the selected structure was divided into 32 cross-sections, and the cross-sectional areas of each slice were integrated to calculate graft volume (MULTI-SLICE method).
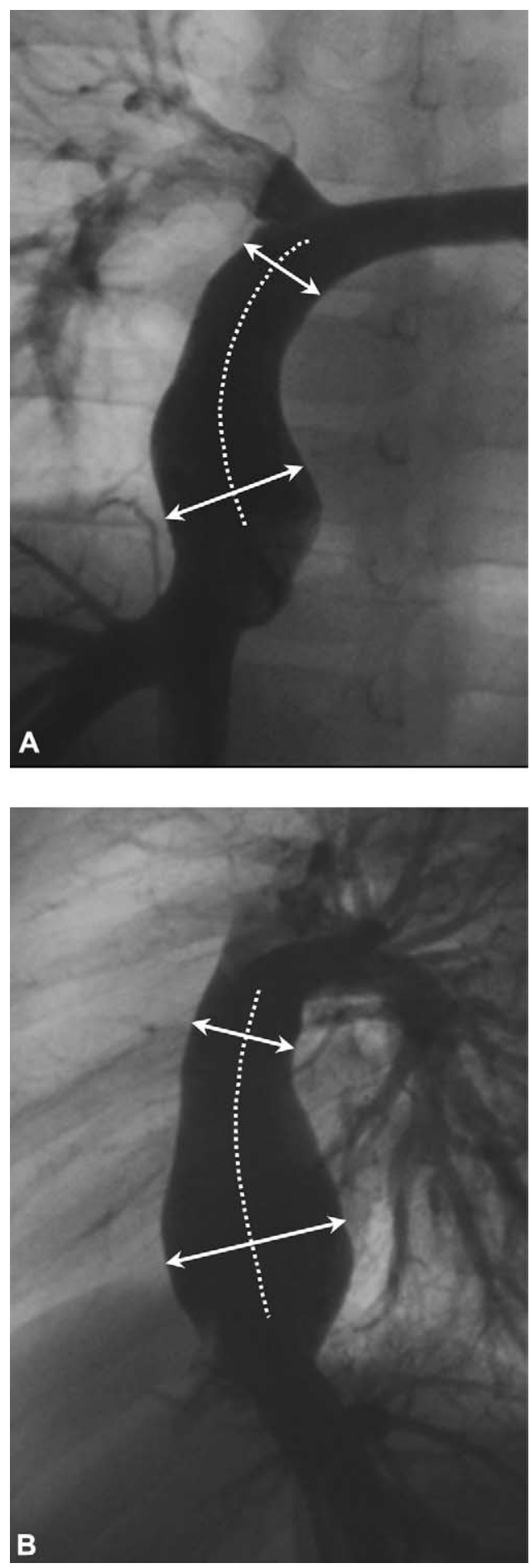

Figure E2. The ratio of the widest to the narrowest diameter in the pedicled autologous pericardial roll conduit was estimated in either the frontal $(A)$ or lateral $(B)$ view to determine proportional change in conduit shape. 